\title{
28 Research Square \\ Prognostic Effect of IncRNA SNHG7 On Cancer Outcome: A Meta and Bioinformatic Analysis
}

\section{yunyuan zhang}

Affiliated Hospital of Qingdao University

\section{Qingwu Tian}

Affiliated Hospital of Qingdao University

\section{Shifeng Huang}

First Affiliated Hospital of Chongqing Medical University

\section{Qing Wang}

Affiliated Hospital of Qingdao University

\section{Hongmei Wu}

Affiliated Hospital of Qingdao University

\section{Qian Dong}

Affiliated Hospital of Qingdao University

xian chen ( $\nabla$ cxkakicoco2014@163.com )

Affiliated Hospital of Qingdao University

\section{Research Article}

Keywords: Cancer, LncRNA, meta-analysis

Posted Date: January 21st, 2021

DOl: https://doi.org/10.21203/rs.3.rs-143840/v1

License: (c) (1) This work is licensed under a Creative Commons Attribution 4.0 International License.

Read Full License

Version of Record: A version of this preprint was published at BMC Cancer on January 3rd, 2022. See the published version at https://doi.org/10.1186/s12885-021-09068-w. 


\section{Abstract}

Background: New evidence from clinical and fundamental researches suggests that SNHG7 is involved in the occurrence and development of carcinomas. And the increase levels of SNHG7 are associated with poor prognosis in various kinds of tumors. However, the small sample size was the limitation for the prognostic value of SNHG7 in clinical application. The aim of the present meta-analysis was to conduct a qualitative analysis to explore the prognostic value of SNHG7 in various cancers.

Methods: Articles related to the SNHG7 as a prognostic biomarker for cancer patients, were comprehensive searched in several electronic databases. The enrolled articles were qualified via the preferred reporting items for systematic reviews and meta-analysis of observational studies in epidemiology checklists. Additionally, an online database based on The Cancer Genome Atlas (TCGA) was further used to validate our results.

Results: We analyzed 2418 cancer patients that met the specified criteria. The present research indicated that an elevated SNHG7 expression level was significantly associated with unfavorable overall survival (OS) (HR $=2.45,95 \% \mathrm{Cl}: 2.12-2.85, p<0.001)$. Subgroup analysis showed that high expression levels of SNHG7 were also significantly associated with unfavorable OS in digestive system cancer $(H R=2.31$, 95\% Cl: 1.90-2.80, $p<0.001)$ and non-digestive system cancer $(\mathrm{HR}=2.67,95 \% \mathrm{Cl}: 2.12-3.37, p<0.001)$. Additionally, increased SNHG7 expression was found to be associated with tumor stage and progression (III/IV vs. I/II: HR $=1.76,95 \%$ Cl: $1.57-1.98, p<0.001$ ). Furthermore, elevated SNHG7 expression significantly predicted lymph node metastasis $(\mathrm{LNM})(\mathrm{HR}=1.98,95 \% \mathrm{Cl}: 1.74-2.26, p<0.001)$ and distant metastasis $(\mathrm{DM})(\mathrm{HR}=2.49,95 \% \mathrm{Cl}: 1.88-3.30, p<0.001)$ respectively. No significant heterogeneity was observed among these studies. SNHG7 was significantly upregulated in four cancers and the elevated expression of SNHG7 predicted shorter OS in four malignancies and worse DFS in five malignancies based on the validation using the GEPIA cohort.

Conclusions: The present analysis suggests that elevated SNHG7 is significantly associated with unfavorable OS, tumor progression, LNM and DM in various carcinomas, and may be used as a promising biomarker to guide therapy for cancer patients.

\section{Background}

With the increasing prevalence of cancer, carcinomas had gradually been recognized as a major threaten to human health the world over $(1,2)$. Although great progresses continued to be made in cancer treatment, it is not satisfactory because the long-term survival rate of many cancers is still remaining very low. With the rapid developments of science and technology, researchers gradually realize that the molecular mechanisms of tumorigenesis and development are still needing further elucidated. Therefore, there is an urgent need to find new and effective clinical biomarkers for early diagnosis, prognosis and ideal therapeutic targets for cancer patients. Long noncoding RNAs (IncRNAs) have a wide range of 
biological functions, such as alternative splicing, chromatin modification, dosage compensation, inactivation of major tumor suppressor genes, and gene imprinting etc.(3-5).

More and more evidences revealed that IncRNAs are deregulated in a variety of carcinomas. It is therefore believed that IncRNAs, with a length of more than 200 nucleotides, may contribute to the development and progression of cancers $(6,7)$. Mounting articles have shown that IncRNAs may be involved in a series of biological pathways including oncogenesis (8). Therefore, as a class of regulatory factors, IncRNAs have attracted extensive attention and may be served as potential biomarkers for carcinomas (9-11).

As a modulator of biological processes, small nucleolus RNA host gene 7 (SNHG7), a long non-coding RNA located on chromosome 9q34.3, is expressed in many cancer tissues. New evidence from clinical and fundamental researches suggests that IncRNA SNHG7 is involved in the occurrence and development of carcinomas. And the increase levels of IncRNA SNHG7 are associated with poor prognosis in various kinds of tumors. However, the small sample size was the limitation for the prognostic value of SNHG7 in clinical application. In the present study, a qualitative meta-analysis was conducted to explore the prognostic effect of SNHG7 on cancer patients.

\section{Methods}

\section{Literature search and selection}

Articles published in English up to Dec $30^{\text {th }}, 2020$, which related to the IncRNA SNHG7 as a prognostic biomarker for cancer patients, were comprehensive searched in several electronic databases. These databases include: Springer, Cochrane Library, Embase, BioMed Central, PubMed, Science Direct and ISI Web of Knowledge. Articles with the following keywords for the online search were included: ("SNHG7" OR "small nuclear RNA host gene 7" OR "Inc RNA-" OR "long noncoding RNA-" OR "noncoding RNA-") AND ("neoplasm" OR "tumor" OR "cancer" OR "carcinoma") AND ("metastasis" OR "prognosis" OR "metastatic" OR "prognostic"). Manually searched of the reference lists were also conducted to obtain potential eligible studies.

\section{Inclusion and exclusion criteria}

Inclusion criteria: 1) definite diagnosis or histopathology confirmed for carcinomas;2) studies investigating the prognostic values of IncRNA SNHG7 in various cancers; 3) sufficient information for computing pooled hazard ratios (HR) and 95\% confidence intervals (CI).

Exclusion criteria: 1) duplicated articles; 2) studies absent of prognostic outcomes; 3) case reports, correspondences, letters, non-human research, review articles and other studies without original data.

\section{Data extraction and quality assessment}

After reviewed the eligible articles, two authors ( $Y Y Z$ and $X C$ ) extracted the necessary data independently. The necessary information from each publication was extracted: (1) last name of first author, publication 
year, country, cancer type, study design, stage, follow-up time and total cases; (2) SNHG7 assessment method and specimen resources; (3) hazard ratio (HR) with 95\% confidence interval (Cl) of SNHG7 for overall survival; (4) patient number for TNM stage and progression, lymph node metastasis and distance metastasis. Preferred reporting items for systematic reviews and meta-analyses (PRISMA) was served to qualified all of the enrolled articles (Supplementary Table 1). Enguage Digitizer (Version 4.1) software was performed to extract HRs with $95 \% \mathrm{Cls}$ from the graphical plots if the eligible literature only provided Kaplan-Meier survival curves as the OS data $(12,13)$.

\section{Validation of bioinformatics database}

Gene Expression Profiling Interactive Analysis (GEPIA), which is based on The Cancer Genome Atlas (TCGA). Survival plots of the correlation between SNHG7 expression and OS or DFS were retrieved as K$M$ curves based on different cancer datasets from GEPIA online database.

\section{Statistical analysis}

The effect of SNHG7 levels on the aggregated overall survival, tumor progression, lymph node metastasis and distance metastasis were evaluated by HRs and $95 \% \mathrm{Cls}$. $P$ statistics was used to calculated heterogeneity among the enrolled studies. The fix-effects model was performed to reveal the relationship between SNHG7 expression levels and clinical outcomes $\left(R_{<} 50 \%\right)(14,15)$. Probable publication bias was evaluated by a funnel plot and Begg's bias test (16). A $p$-value $<0.05$ was regarded as significantly statistical. All analyses were conducted with RevMan 5.3 software and Stata SE 12.0 (Stata Corporation).

\section{Results}

\section{Included articles}

Literature screening and study selection processes were presented as Fig 1 . The preliminary online search retrieved 548 publications concerning the prognosis or metastasis of SNHG7 and cancer patients. After carefully removing the duplicates, 28 articles were excluded and 408 publications proceed to abstract screening. We then carefully remove another 385 studies according to the inclusion and exclusion criteria. Finally, 23 articles were enrolled for the meta-analysis in this study(17-39).

\section{Characteristics of the enrolled studies}

Table 1 summarized the main characteristics of the enrolled twenty-three eligible articles. All of the 2418 participants were from China and divided into high or low group according to the qRT-PCR or microarray results. The cut-off values were different, with median was applied in most articles. Nineteen of the enrolled studies investigated the expression level of SNHG7 and overall survival. Twenty-two articles were associated with the level of SNHG7 and tumor progression or metastasis.

\section{Meta-analysis results}


Finally, nineteen studies were enrolled to analyze IncRNA SNHG7 expression levels and cancer patient outcomes. A fix-effects model was conducted to calculate the pooled effect size because no significant heterogeneity was existed among the enrolled studies $(R=0 \%)$. Our results revealed that the increased SNHG7 was significantly related to the unfavorable OS (HR $=2.45,95 \% \mathrm{Cl}: 2.12-2.85, p<0.001)$ (Fig. 2). Subgroup analysis showed that high expression levels of SNHG7 were also significantly associated with unfavorable OS in digestive system cancer patients (HR $=2.31,95 \% \mathrm{Cl}: 1.90-2.80, p<0.001)$ and nondigestive system cancer patients ( $\mathrm{HR}=2.67,95 \% \mathrm{Cl}: 2.12-3.37, p<0.001)$ (Fig. 3).

Additionally, increased SNHG7 expression was found to be associated with tumor stage and progression (III/IV vs. I/II: HR $=1.76,95 \% \mathrm{Cl}: 1.57-1.98, p<0.001$ ) (Fig. 4). Furthermore, elevated SNHG7 expression significantly predicted lymph node metastasis $(\mathrm{LNM})(\mathrm{HR}=1.98,95 \% \mathrm{Cl}: 1.74-2.26, p<0.001)$ and distant metastasis $(\mathrm{DM})(\mathrm{HR}=2.49,95 \% \mathrm{Cl}: 1.88-3.30, p<0.001)$ respectively (Fig. $5 \mathrm{~A}$ and $5 \mathrm{~B})$.

\section{Publication bias}

Publication bias of the nineteen studies in this analysis was assessed by funnel plot and Begg's bias test. The shape of the funnel plot was symmetrical and the Begg's test revealed that no significant publication bias was existed ( $p>0.05)$ (Fig. 6A).

\section{Sensitivity analysis}

Through sensitivity analysis of these nineteen enrolled articles, it was indicated that the pooled SNHG7 HR was not significantly affected by the exclusion of any single study (Fig. 6B).

\section{Validation of the results in the GEPIA database}

The GEPIA database was used to further validate our results. In terms of SNHG7 dysregulation, SNHG7 overexpression was identified in Cholangiocarcinoma (CHOL), Lymphoid Neoplasm Diffuse Large B-cell Lymphoma (DLBC), Pheochromocytoma and Paraganglioma (PCPG), and thymoma (THYM) (Fig. 7). Regarding the association between SNHG7 expression and prognosis, increased SNHG7 expression was correlated with worse OS in Adrenocortical carcinoma (ACC), Colon adenocarcinoma (COAD), Mesothelioma (MESO), Uterine Carcinosarcoma (UCS) and with worse DFS in Adrenocortical carcinoma (ACC), Kidney renal papillary cell carcinoma (KIRP), Liver hepatocellular carcinoma (LIHC), Lung squamous cell carcinoma (LUSC), Uterine Carcinosarcoma (UCS) (log-rank $\mathrm{P}<0.05$ ) (Fig. 8 and 9). These results support our results and indicate that SNHG7 could be a novel prognostic biomarker for various cancers.

\section{Discussion}

Early scientists believed that IncRNAs are transcriptional noises due to the fact that most IncRNAs are generated by intron and intergenic regions of the genomes, and lack of protein coding capacity. In recent years, scientists have gradually discovered that IncRNAs may regulate the expression of target genes, 
involve in biological processes, and may be acted as oncogenes or tumor suppressors. With the rapid expansion of high throughput genomic sequencing technology, IncRNAs have been proved to be deregulated in various tumors, and even to be used as promising prognostic biomarkers in cancer patients. Many clinical and fundamental studies suggested that increasing levels of SNHG7 have intimate terms with unfavorable prognosis and progression in cancer patients. However, the small sample size was the limitation for the prognostic value of SNHG7 in clinical application. As far as we know, no systematic meta-analysis has been performed on SNHG7 expression levels and various cancer patients' outcomes.

LncRNA SNHG7, a novel discovered IncRNA located on chromosome 9q34.3, and has been proved to be significantly up-regulated in various carcinomas, such as bladder cancer, breast cancer, cervical cancer, colorectal cancer, gastric cancer, hepatocellular carcinoma, hypo pharyngeal cancer, melanoma, neuroblastoma, non-small cell lung cancer, pancreatic cancer, prostate cancer, thyroid cancer etc. These aberrant patterns of expression were associated with specific clinical features, such as overall survival time, lymph node metastasis, distant metastasis and TNM stage. SNHG7 serves as an oncogene and contributes to cell biological functions in various cancers, which including apoptosis inhibition, cell proliferation, cell cycle arrest, invasion, migration, and vasculogenic mimicry. Furthermore, SNHG7 may act as a competitive endogenous RNA (ceRNA) to aggravate the development of cancers. Elevated IncRNA SNHG7 may reduce the miRNAs expression level, such as miR34a, miR3243p, miR503, miRNA381, miR186, miR216b and miR5095 in multiple cancers $(25,26,40-44)$. Taken together, these articles demonstrated that SNHG7 plays an important role in tumor development and progression. These evidences encouraged us to investigate the correlation between SNHG7 expression levels and cancer prognosis. And our results demonstrated that elevated IncRNA SNHG7 is an unfavorable predictor for various cancer patients.

Twenty-three published studies that included 2418 patients were enrolled in this analysis. Our results revealed that the increased SNHG7 was significantly related to the unfavorable OS ( $\mathrm{HR}=2.45,95 \% \mathrm{Cl}$ : $2.12-2.85, p<0.001)$. Subgroup analysis showed that high expression levels of SNHG7 were also significantly associated with unfavorable $\mathrm{OS}$ in digestive system cancer $(\mathrm{HR}=2.31,95 \% \mathrm{Cl}: 1.90-2.80, p$ $<0.001)$ and non-digestive system cancer ( $\mathrm{HR}=2.67,95 \% \mathrm{Cl}: 2.12-3.37, p<0.001)$. Additionally, increased SNHG7 expression was found to be associated with tumor stage and progression (III/IV vs. I/II: $\mathrm{HR}=1.76,95 \% \mathrm{Cl}: 1.57-1.98, p<0.001)$. Furthermore, elevated SNHG7 expression significantly predicted lymph node metastasis (LNM) (HR $=1.98,95 \% \mathrm{Cl}: 1.74-2.26, p<0.001)$ and distant metastasis (DM) (HR $=2.49,95 \% \mathrm{Cl}: 1.88-3.30, \mathrm{p}<0.001)$ respectively. GEPIA database was further used to validate our results as broadly as possible. High SNHG7 expression levels were observed in CHOL, DLBC, PCPG and THYM. What's more, increased SNHG7 expression was correlated with worse OS in ACC, COAD, MESO, UCS and with worse DFS in ACC, KIRP, LUSC, UCS. Taken together, these results indicate that SNHG7 could be a novel prognostic biomarker for various cancers.

The present meta-analysis has limitations that only the researches published in English were included. Next, this study was constrained to studies published in China, so our results may best illustrate the 
association between SNHG7 and Asian patients. Well-designed studies and multi-ethnic clinical researches with larger sample size should be carried out in the future. Third, some HRs are extracted by reconstructing the K-M curve, rather than directly from the original research, which would inevitably lead to possible deviations. Despite the inherent deficiencies, our study provides strong evidence that elevated IncRNA SNHG7 expression levels are prognostic for reduced OS, tumor progression, LYM and DM in cancer patients.

\section{Conclusion}

In conclusion, the present analysis implicated that elevated SNHG7 is strongly associated with OS, tumor progression, LNM and DM in carcinomas, and may be used as a promising biomarker to guide therapy for various cancer patients.

\section{Abbreviations}

$95 \% \mathrm{Cl}$

95\% confidence interval; ceRNA:Competing endogenous RNA;EFS:Event free survival; HR:Hazard ratio; LncRNA:Long noncoding RNA;OS:Overall survival; PFS:Progress free survival; RFS:Relapse free survival;SNHG1:Small nucleolar RNA host gene 1

\section{Declarations}

\section{Author contribution}

Conceived and designed the experiments: X.C, Y.Y.Z and Q.D. Performed the experiments: X.C., Y.Y.Z., Q.W.T.,SFH.,HMW and Q.D. Analyzed the data: X.C., Q.W., Q.W.T., Y.Y.Z. Contributed reagents/materials/analysis tools: X.C., Q.D.; Y.Y.Z. Wrote the paper: X.C., Y.Y.Z.

\section{Funding}

This study is fully supported by the National Natural Science Foundation of China (No. 81601821 to YYZ), Livelihood Science and technology program of Qingdao (No.17-3-3-8-nsh to QD), Shandong Key Laboratory of Digital Medicine and Computer-Assisted Surgery Foundation (to QD).

\section{Availability of data and materials}

All data analyzed during this study are included in this published article. GEPIA database is publicly available at $\underline{h t t p: / / g e p i a . c a n c e r-p k u . c n / i n d e x . h t m l .}$

\section{Ethics approval and consent to participate}

Not applicable. 


\section{Consent for publication}

Not applicable.

\section{Competing interests}

The authors declare that they have no competing interests.

\section{Acknowledgements}

We are grateful to all researchers of enrolled studies.

\section{References}

1. Bray F, Ren JS, Masuyer E, Ferlay J. Global estimates of cancer prevalence for 27 sites in the adult population in 2008. Int J Cancer. 2013;132(5):1133-45.

2. Siegel RL, Miller KD, Jemal A. Cancer statistics, 2018. CA Cancer J Clin. 2018;68(1):7-30.

3. Moran VA, Perera RJ, Khalil AM. Emerging functional and mechanistic paradigms of mammalian long non-coding RNAs. Nucleic Acids Res. 2012;40(14):6391-400.

4. Tian D, Sun S, Lee JT. The long noncoding RNA, Jpx, is a molecular switch for X chromosome inactivation. Cell. 2010;143(3):390-403.

5. Tsai MC, Spitale RC, Chang HY. Long intergenic noncoding RNAs: new links in cancer progression. Cancer Res. 2011;71(1):3-7.

6. Poliseno L, Salmena L, Zhang J, Carver B, Haveman WJ, Pandolfi PP. A coding-independent function of gene and pseudogene mRNAs regulates tumour biology. Nature. 2010;465(7301):1033-8.

7. Gibb EA, Brown CJ, Lam WL. The functional role of long non-coding RNA in human carcinomas. Mol Cancer. 2011;10:38.

8. Ji J, Xu J, Zhao S, Liu F, Qi J, Song Y, et al. Myeloid-derived suppressor cells contribute to systemic lupus erythaematosus by regulating differentiation of Th17 cells and Tregs. Clin Sci (Lond). 2016;130(16):1453-67.

9. Jing X, Lin J, Wang H, Tian L, Tian R, Zhang Y, et al. Prognostic value of IncRNA SOX2OT for Chinese cancer patients: A meta-analysis. PLoS One. 2017;12(5):e0176889.

10. Zhang Y, Lun L, Li H, Wang Q, Lin J, Tian R, et al. The Value of IncRNA NEAT1 as a Prognostic Factor for Survival of Cancer Outcome: A Meta-Analysis. Sci Rep. 2017;7(1):13080.

11. Chen X, Lun L, Hou H, Tian R, Zhang H, Zhang Y. The Value of IncRNA HULC as a Prognostic Factor for Survival of Cancer Outcome: A Meta-Analysis. Cell Physiol Biochem. 2017;41(4):1424-34.

12. Parmar MK, Torri V, Stewart L. Extracting summary statistics to perform meta-analyses of the published literature for survival endpoints. Stat Med. 1998;17(24):2815-34.

13. Tierney JF, Stewart LA, Ghersi D, Burdett S, Sydes MR. Practical methods for incorporating summary time-to-event data into meta-analysis. Trials. 2007;8:16. 
14. Higgins JP, Thompson SG. Quantifying heterogeneity in a meta-analysis. Stat Med. 2002;21(11):1539-58.

15. Bowden J, Tierney JF, Copas AJ, Burdett S. Quantifying, displaying and accounting for heterogeneity in the meta-analysis of RCTs using standard and generalised Q statistics. BMC Med Res Methodol. 2011;11:41.

16. Begg CB, Mazumdar M. Operating characteristics of a rank correlation test for publication bias. Biometrics. 1994;50(4):1088-101.

17. Chen $Y$, Peng $Y, X u Z$, , Ge B, Xiang X, Zhang T, et al. Knockdown of IncRNA SNHG7 inhibited cell proliferation and migration in bladder cancer through activating Wnt/beta-catenin pathway. Pathol Res Pract. 2019;215(2):302-7.

18. Cheng D, Fan J, Ma Y, Zhou Y, Qin K, Shi M, et al. LncRNA SNHG7 promotes pancreatic cancer proliferation through ID4 by sponging miR-342-3p. Cell Biosci. 2019;9:28.

19. Chi R, Chen X, Liu M, Zhang H, Li F, Fan X, et al. Role of SNHG7-miR-653-5p-STAT2 feedback loop in regulating neuroblastoma progression. J Cell Physiol. 2019;234(8):13403-12.

20. Hu Y, Wang L, Li Z, Wan Z, Shao M, Wu S, et al. Potential Prognostic and Diagnostic Values of CDC6, CDC45, ORC6 and SNHG7 in Colorectal Cancer. Onco Targets Ther. 2019;12:11609-21.

21. Jia J, Zhang D, Zhang J, Yang L, Zhao G, Yang H, et al. Long non-coding RNA SNHG7 promotes neuroblastoma progression through sponging miR-323a-5p and miR-342-5p. Biomed Pharmacother. 2020;128:110293.

22. Jiang $D$, Wang $C, \mathrm{He} J$. Long non-coding RNA DGCR5 incudes tumorigenesis of triple-negative breast cancer by affecting Wnt/beta-catenin signaling pathway. J BUON. 2020;25(2):702-8.

23. Li Y, Zeng C, Hu J, Pan Y, Shan Y, Liu B, et al. Long non-coding RNA-SNHG7 acts as a target of miR34a to increase GALNT7 level and regulate PI3K/Akt/mTOR pathway in colorectal cancer progression. J Hematol Oncol. 2018;11(1):89.

24. Pang L, Cheng Y, Zou S, Song J. Long noncoding RNA SNHG7 contributes to cell proliferation, migration, invasion and epithelial to mesenchymal transition in non-small cell lung cancer by regulating miR-449a/TGIF2 axis. Thorac Cancer. 2020;11(2):264-76.

25. Qi H, Wen B, Wu Q, Cheng W, Lou J, Wei J, et al. Long noncoding RNA SNHG7 accelerates prostate cancer proliferation and cycle progression through cyclin D1 by sponging miR-503. Biomed Pharmacother. 2018;102:326-32.

26. Shan Y, Ma J, Pan Y, Hu J, Liu B, Jia L. LncRNA SNHG7 sponges miR-216b to promote proliferation and liver metastasis of colorectal cancer through upregulating GALNT1. Cell Death Dis. 2018;9(7):722.

27. Shen A, Ma J, Hu X, Cui X. High expression of IncRNA-SNHG7 is associated with poor prognosis in hepatocellular carcinoma. Oncol Lett. 2020;19(6):3959-63.

28. Wang YH, Huo BL, Li C, Ma G, Cao W. Knockdown of long noncoding RNA SNHG7 inhibits the proliferation and promotes apoptosis of thyroid cancer cells by downregulating BDNF. Eur Rev Med Pharmacol Sci. 2019;23(11):4815-21. 
29. Wang W, Liu G, Liu M, Li X. Long non-coding RNA SNHG7 promotes malignant melanoma progression through negative modulation of miR-9. Histol Histopathol. 2020;35(9):973-81.

30. Wu P, Tang Y, Fang X, Xie C, Zeng J, Wang W, et al. Metformin Suppresses Hypopharyngeal Cancer Growth by Epigenetically Silencing Long Non-coding RNA SNHG7 in FaDu Cells. Front Pharmacol. 2019;10:143.

31. Wu F, Sui Y, Wang Y, Xu T, Fan L, Zhu H. Long Noncoding RNA SNHG7, a Molecular Sponge for microRNA-485, Promotes the Aggressive Behavior of Cervical Cancer by Regulating PAK4. Onco Targets Ther. 2020;13:685-99.

32. Xia Q, Li J, Yang Z, Zhang D, Tian J, Gu B. Long non-coding RNA small nucleolar RNA host gene 7 expression level in prostate cancer tissues predicts the prognosis of patients with prostate cancer. Medicine (Baltimore). 2020;99(7):e18993.

33. Xie Y, Wang Y, Gong R, Lin J, Li X, Ma J, et al. SNHG7 Facilitates Hepatocellular Carcinoma Occurrence by Sequestering miR-9-5p to Upregulate CNNM1 Expression. Cancer Biother Radiopharm. 2020.

34. Yang X, Sun L, Wang L, Yao B, Mo H, Yang W. LncRNA SNHG7 accelerates the proliferation, migration and invasion of hepatocellular carcinoma cells via regulating miR-122-5p and RPL4. Biomed Pharmacother. 2019;118:109386.

35. Zeng J, Ma YX, Liu ZH, Zeng YL. LncRNA SNHG7 contributes to cell proliferation, invasion and prognosis of cervical cancer. Eur Rev Med Pharmacol Sci. 2019;23(21):9277-85.

36. Zhang Y, Yuan Y, Zhang Y, Cheng L, Zhou X, Chen K. SNHG7 accelerates cell migration and invasion through regulating miR-34a-Snail-EMT axis in gastric cancer. Cell Cycle. 2020;19(1):142-52.

37. Zhang P, Shi L, Song L, Long Y, Yuan K, Ding W, et al. LncRNA CRNDE and IncRNA SNHG7 are Promising Biomarkers for Prognosis in Synchronous Colorectal Liver Metastasis Following Hepatectomy. Cancer Manag Res. 2020;12:1681-92.

38. Zhao D, Zhang H, Long J, Li M. LncRNA SNHG7 Functions as an Oncogene in Cervical Cancer by Sponging miR-485-5p to Modulate JUND Expression. Onco Targets Ther. 2020;13:1677-89.

39. Zhong X, Long Z, Wu S, Xiao M, Hu W. LncRNA-SNHG7 regulates proliferation, apoptosis and invasion of bladder cancer cells assurance guidelines. J BUON. 2018;23(3):776-81.

40. Deng Y, Zhao F, Zhang Z, Sun F, Wang M. Long Noncoding RNA SNHG7 Promotes the Tumor Growth and Epithelial-to-Mesenchymal Transition via Regulation of miR-34a Signals in Osteosarcoma. Cancer Biother Radiopharm. 2018;33(9):365-72.

41. Han Y, Hu H, Zhou J. Knockdown of LncRNA SNHG7 inhibited epithelial-mesenchymal transition in prostate cancer though miR-324-3p/WNT2B axis in vitro. Pathol Res Pract. 2019;215(10):152537.

42. Gao YT, Zhou YC. Long non-coding RNA (IncRNA) small nucleolar RNA host gene 7 (SNHG7) promotes breast cancer progression by sponging miRNA-381. Eur Rev Med Pharmacol Sci. 2019;23(15):6588-95.

43. Zhang H, Zhang XY, Kang XN, Jin LJ, Wang ZY. LncRNA-SNHG7 Enhances Chemotherapy Resistance and Cell Viability of Breast Cancer Cells by Regulating miR-186. Cancer Manag Res. 2020;12:10163- 
72.

44. Ren J, Yang Y, Xue J, Xi Z, Hu L, Pan SJ, et al. Long noncoding RNA SNHG7 promotes the progression and growth of glioblastoma via inhibition of miR-5095. Biochem Biophys Res Commun. 2018;496(2):712-8.

\section{Tables}

Due to technical limitations, table 1 is only available as a download in the Supplemental Files section.

Figures 


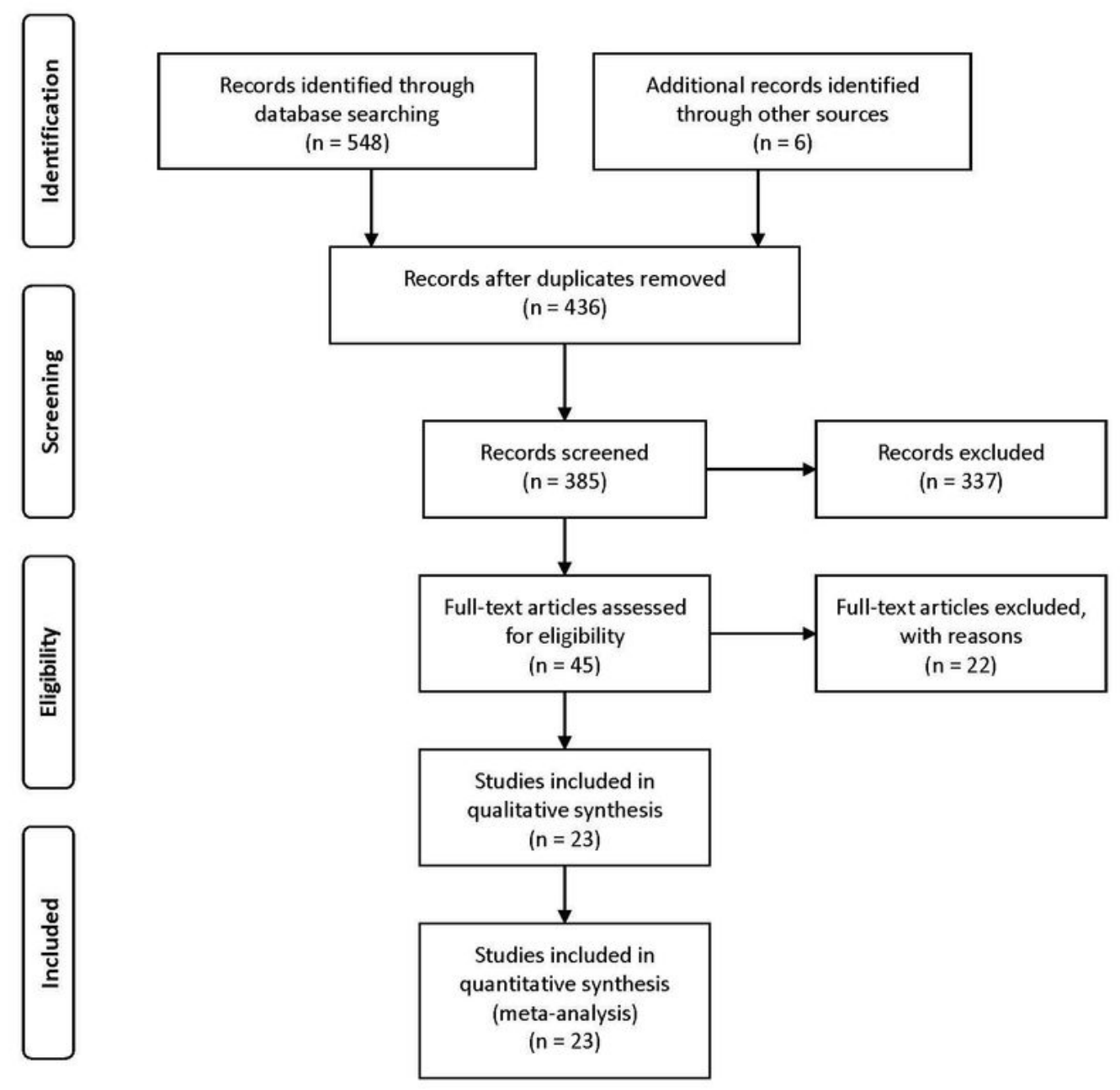

\section{Figure 1}

Flow diagram of the study search and selection process. 
Study

ID

ES $(95 \% \mathrm{Cl}) \quad$ Weight

Chen 2018

Cheng 2019

Chi 2018

Hu 2019

Jia 2020

Li 2018

Qi 2018

Shan 2018

Shen 2020

Wang 2020

Wu 2019

Wu 2020

Xia 2019

Xie 2020

Yang 2019

Zeng 2019

Zhang 2019

Zhang 2020

Zhao 2020

Overall $(\mathrm{I}$-squared $=0.0 \%, p=0.994$ )

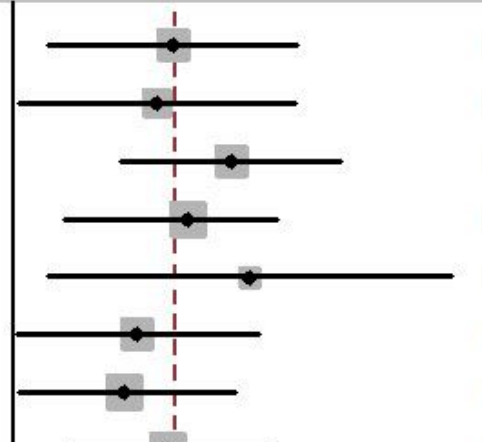

$2.43(1.22,4.83) \quad 4.62$

$2.22(1.03,4.79) \quad 3.71$

$3.35(1.81,6.17) \quad 5.82$

$2.63(1.33,4.32) \quad 6.28$

$3.70(1.21,11.32) \quad 1.75$

$1.99(1.02,3.89) \quad 4.89$

$1.86(1.04,3.42) \quad 6.18$

$2.37(1.32,4.21) \quad 6.51$

$1.85(1.05,3.26) \quad 6.82$

$2.65(1.37,5.24) \quad 4.86$

$2.88(1.48,5.59) \quad 4.96$

$2.64(1.07,6.48) \quad 2.70$

$2.84(1.92,8.38) \quad 4.03$

$2.53(1.04,3.27) \quad 6.67$

$2.60(1.45,4.65) \quad 6.45$

$4.43(1.63,12.07) \quad 2.18$

$2.11(1.61,3.75) \quad 12.24$

$2.87(1.13,4.11) \quad 5.25$

$2.19(1.05,4.54) \quad 4.08$

$2.45(2.12,2.85) \quad 100.00$

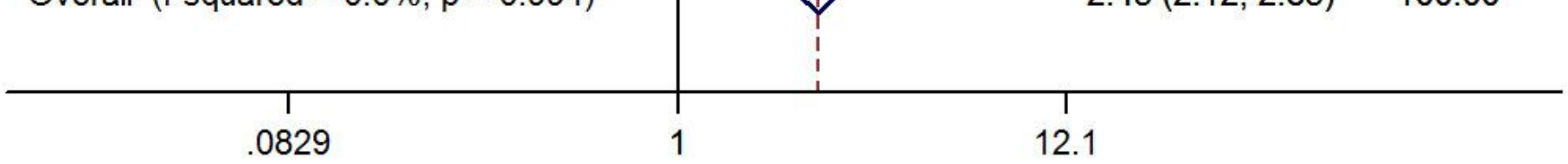

Figure 2

Forest plot of enrolled studies for the association between SNHG7 expression levels with overall survival (OS). 


$\begin{array}{llll}\text { Study } & & & \\ \text { ID } & \text { WS }(95 \% \mathrm{CI}) & \\ \text { Weight }\end{array}$

Figure 3

Stratified analysis by factor of cancer type for the association between SNHG7 expression with OS. 
III/N

Cheng 2019
Chi 2018

Jia 2020

Jiang 2020

Li 2018

Pang 2020

Shan 2018

Shen 2020

Wang 2019

Wang 2020

Wu 2019

Wu 2020

Xia 2019

Zeng 2019

Zhang 2019

Zhao 2020

Total $(\mathbf{9 5} \% \mathrm{Cl})$

Total events

Heterogeneity: $\mathrm{Chi}^{2}=9.15, \mathrm{df}=15(\mathrm{P}=0.87) ; \mathrm{I}^{2}=0 \%$

Test for overall effect: $Z=9.55(P \leq 0.00001)$

$23 \quad 29$

$59 \quad 103$

$10 \quad 18$

22

8 226
Risk Ratio

Total Weight M-H, Fixed, 95\% Cl

$4.8 \% \quad 1.61[1.02,2.53]$

$\begin{array}{llllll}33 & 48 & 20 & 44 & 9.5 \% & 1.51[1.04,2.20]\end{array}$

$\begin{array}{lllll}14 & 17 & 11 & 28 & 3.8 \%\end{array}$

$\begin{array}{lllll}18 & 25 & 13 & 32 & 5.2 \%\end{array}$

$\begin{array}{lllll}21 & 27 & 9 & 26 & 4.2 \%\end{array}$

$\begin{array}{lllll}17 & 22 & 5 & 20 & 2.4 \%\end{array}$

$\begin{array}{lllll}21 & 27 & 9 & 21 & 4.6 \%\end{array}$

$\begin{array}{lllll}23 & 33 & 30 & 67 & 9.1 \%\end{array}$

$\begin{array}{lllll}26 & 36 & 10 & 28 & 5.1 \%\end{array}$

$\begin{array}{lllll}30 & 44 & 16 & 36 & 8.1 \%\end{array}$

$\begin{array}{lllll}30 & 42 & 15 & 31 & 7.9 \%\end{array}$

$\begin{array}{lllll}17 & 24 & 9 & 27 & 3.9 \%\end{array}$

$\begin{array}{lllll}37 & 56 & 29 & 71 & 11.7 \%\end{array}$

31

$59 \quad 12.8 \%$

$27 \quad 2.9 \%$

$2.10[1.26,3.49]$

$1.77[1.09,2.88]$

$2.25[1.28,3.96]$

$3.09[1.40,6.83]$

$1.81[1.06,3.09]$

$1.56[1.10,2.21]$

$2.02[1.18,3.46]$

$1.53[1.01,2.33]$

$1.48[0.98,2.23]$

$2.13[1.18,3.84]$

$1.62[1.15,2.27]$

$2.73[1.53,4.88]$

$1.54[1.06,2.23]$

$1.88[0.92,3.83]$

$1.76[1.57,1.98]$

$569100.0 \%$

(1)

\section{Figure 4}

Forest plot of enrolled studies for the association between SNHG7 expression levels with TNM stage (III/IV vs. I/II). 


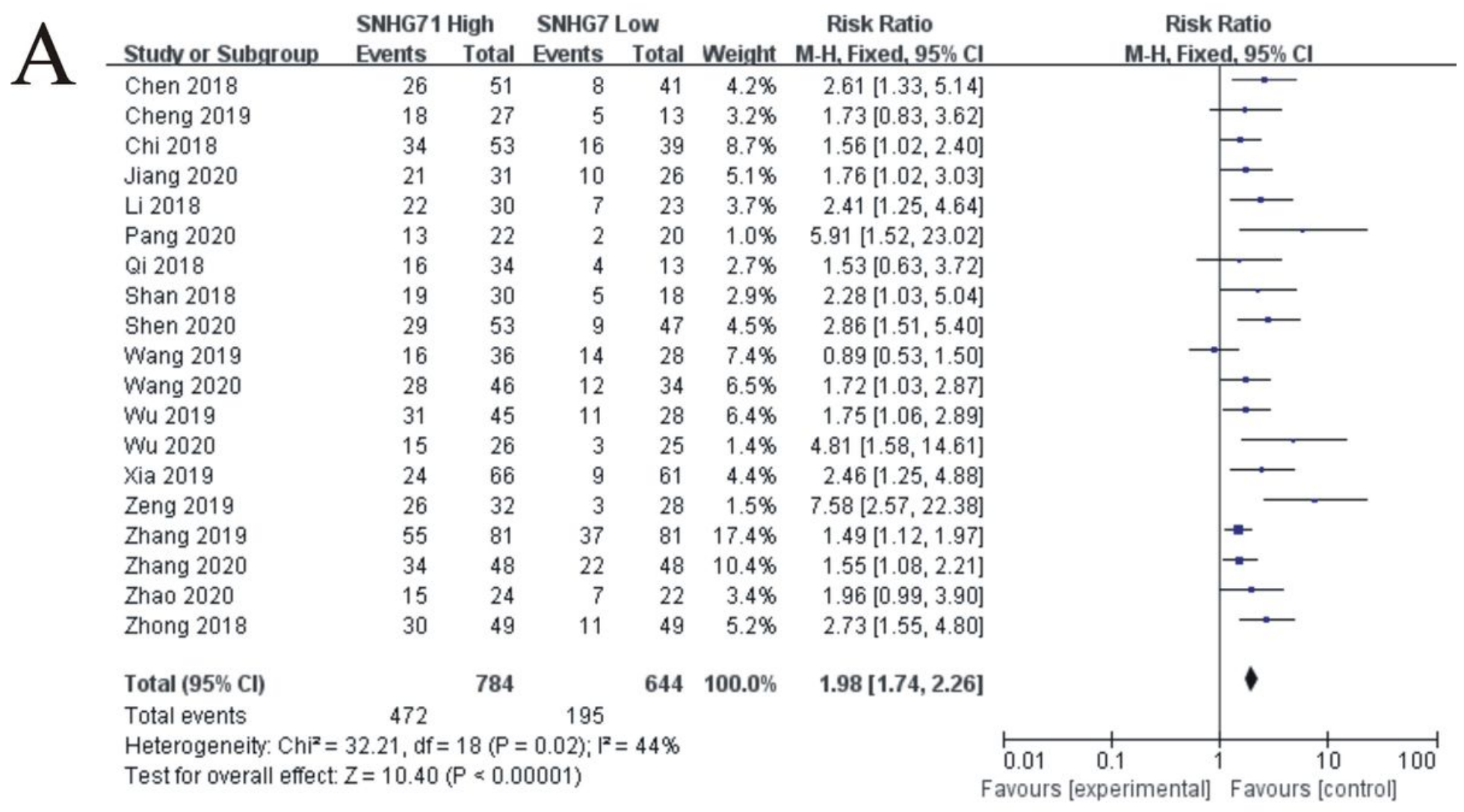

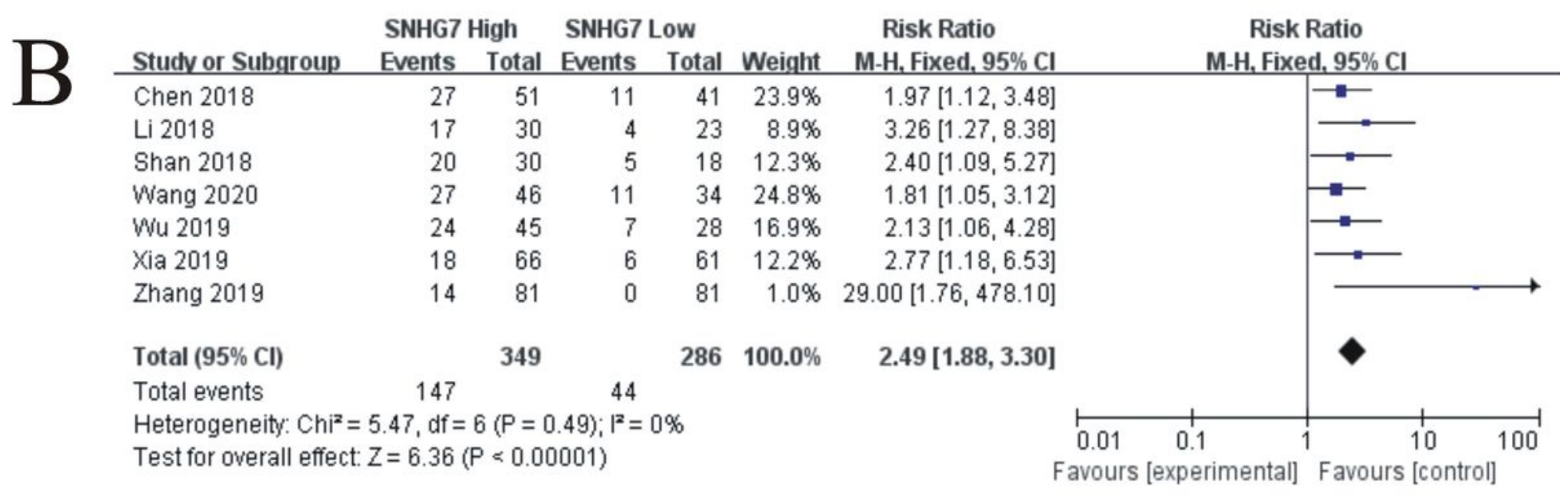

\section{Figure 5}

A. Forest plot for the association between SNHG7 expression levels with LNM. B. Forest plot for the association between SNHG7 expression levels with DM. 


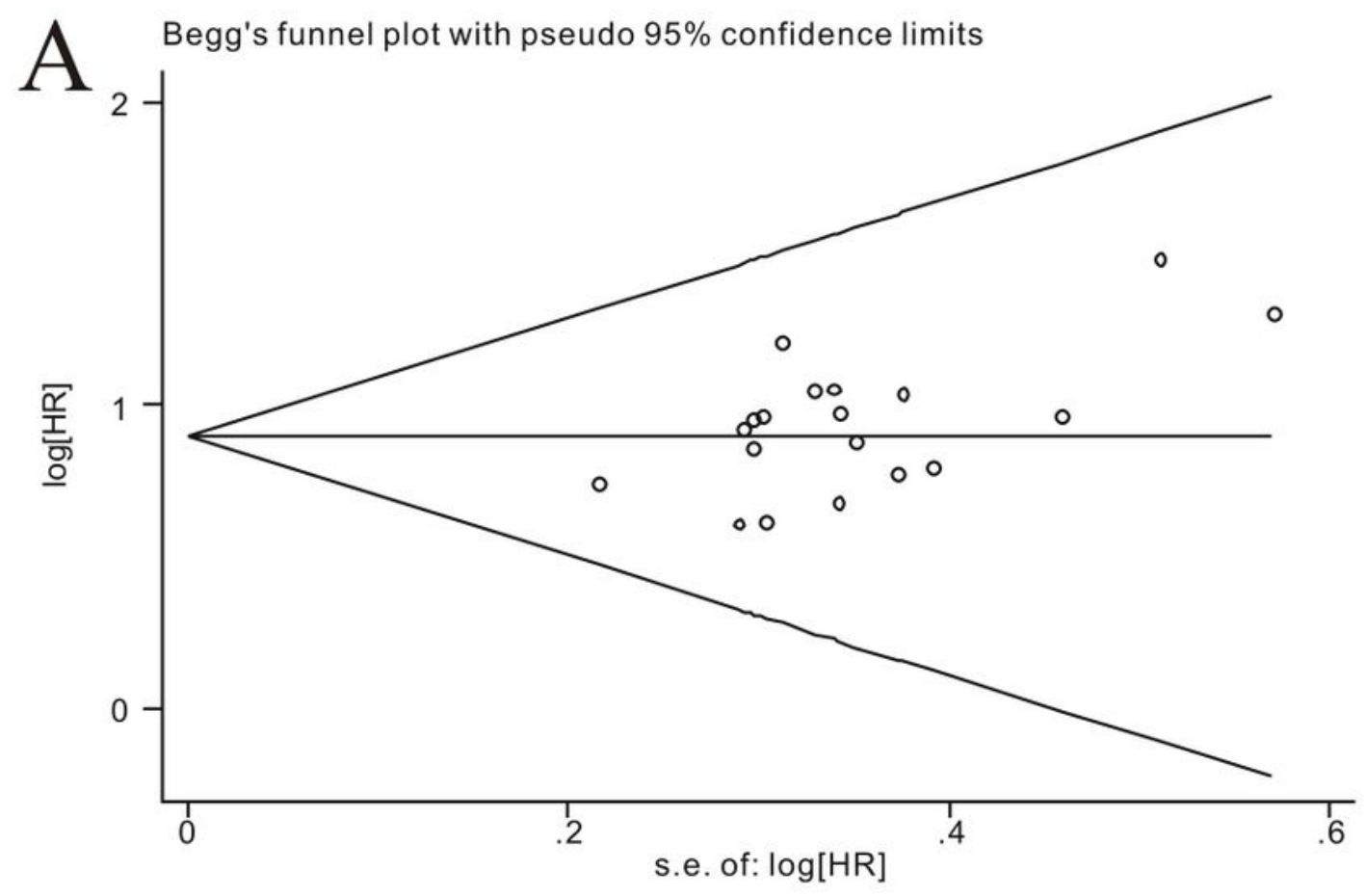

$\mathrm{B}$ Meta-analysis fixed-effects estimates (exponential form) Study ommited

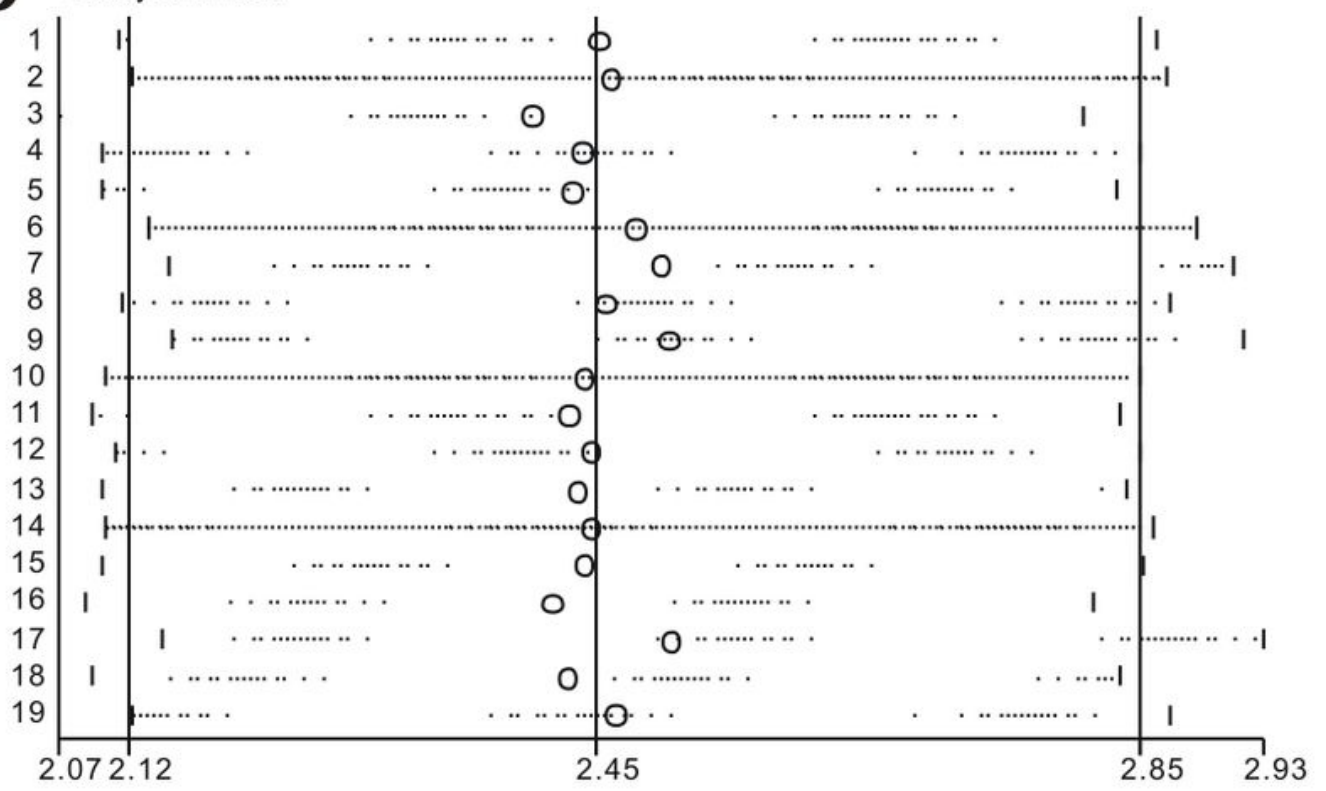

\section{Figure 6}

A. Begg's funnel plot of publication bias for overall survival. B. Sensitivity analysis of ten studies concerning SNHG7 and overall survival. 
A

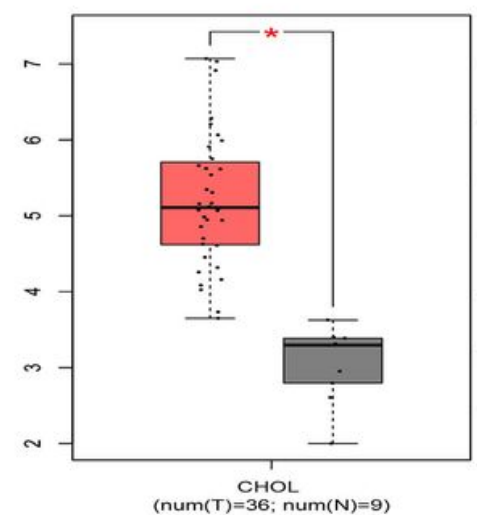

B

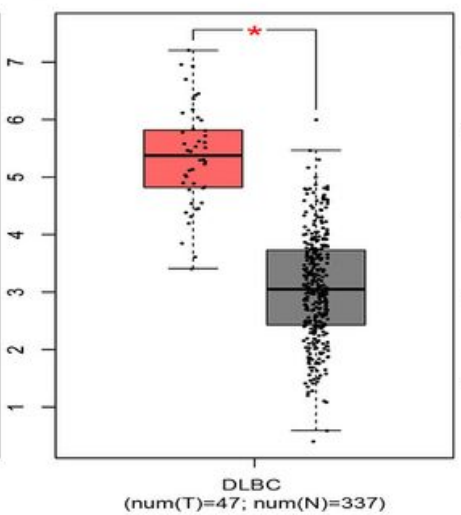

C

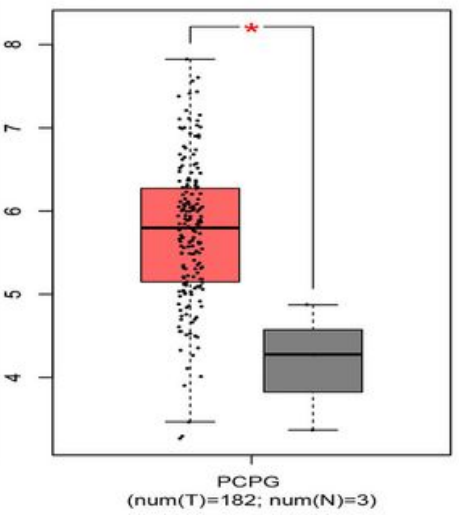

D

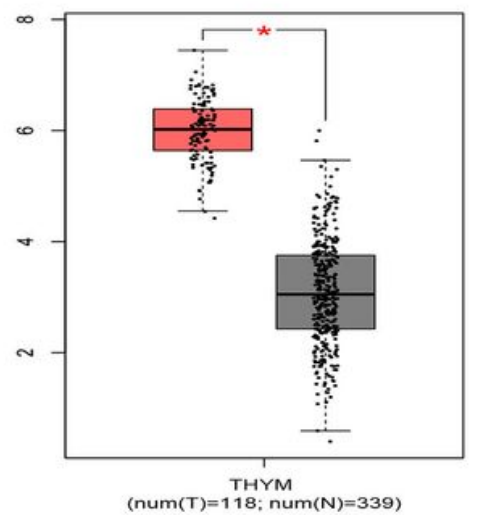

Figure 7

SNHG7 expression in four types of cancer vs. normal tissue. “*” $\mathrm{LLog} 2$ Fold Change $\mathbb{\text { }}>1$ and $p<0.01$. The red box plots represent SNHG7 expression in cancer tissues and the grey box plots represent SNHG7 expression in normal tissues.
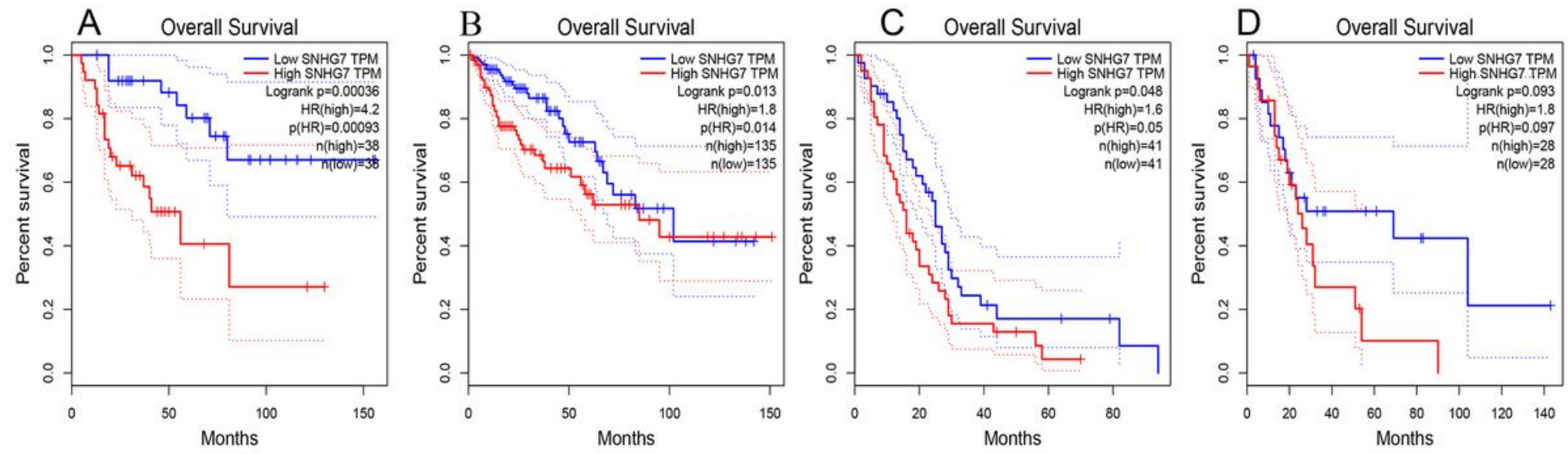

Figure 8

Validation of the prognostic effect of SNHG7 on cancer patient OS based on the TCGA online database. A. OS plot of LUCAT1 in ACC. B. OS plot of LUCAT1 in COAD. C. OS plot of LUCAT1 in MESO. D. OS plot of LUCAT1 in UCS. 

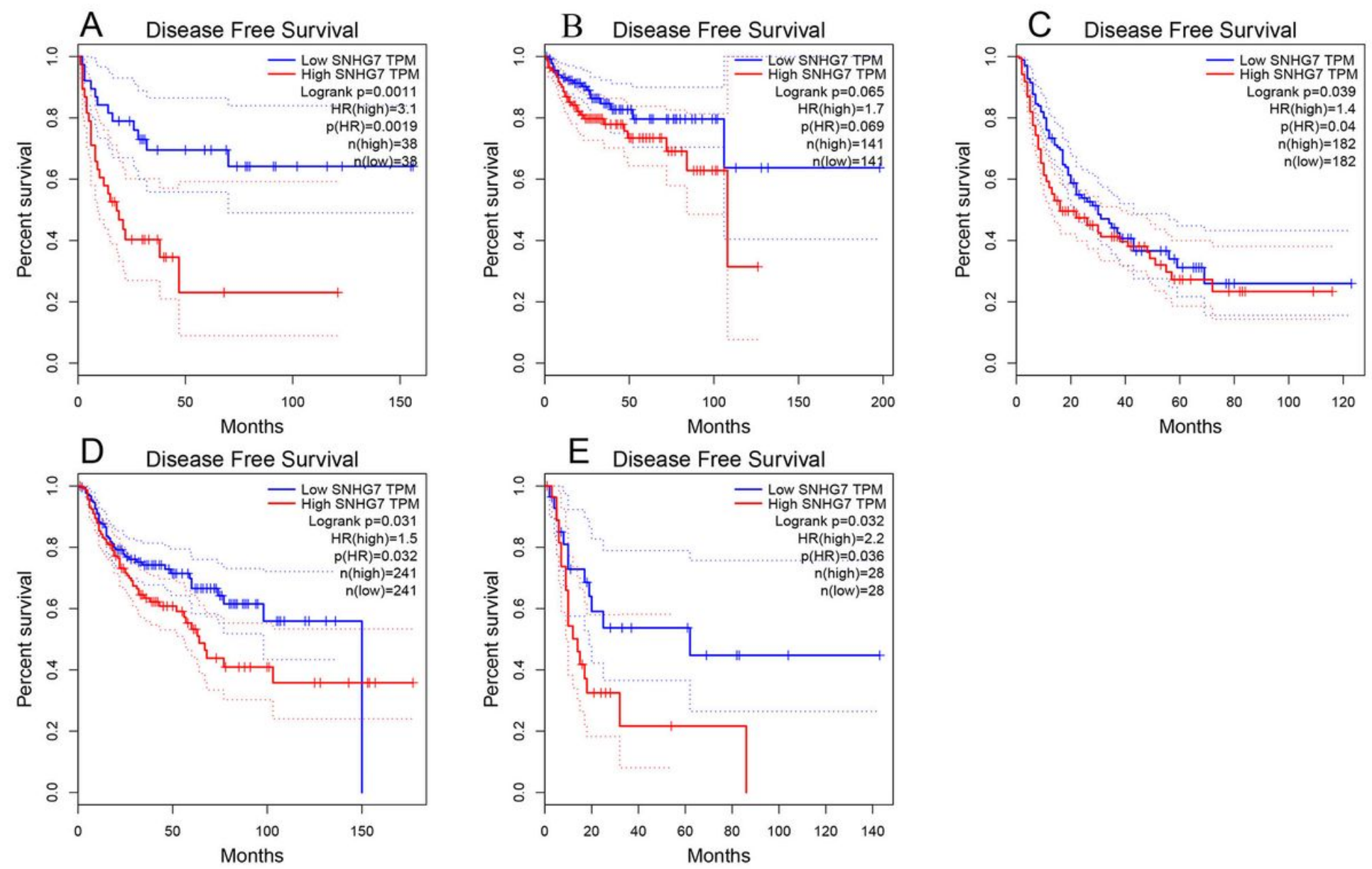

Figure 9

Validation of the prognostic effect of SNHG7 on cancer patient DFS based on the TCGA online database. A. DFS plot of LUCAT1 in ACC. B. DFS plot of LUCAT1 in KIRP. C. DFS plot of LUCAT1 in LIHC. D. DFS plot of LUCAT1 in LUSC. E. DFS plot of LUCAT1 in UCS.

\section{Supplementary Files}

This is a list of supplementary files associated with this preprint. Click to download.

- TableS1.PRISMAchecklist.doc

- TableS2.MOOSEchecklist.docx

- Table1.SNHG7summary.pdf 